\title{
Bases de la crisis del parlamentarismo en Italia y España
}

\author{
Gabriele Ranzato
}

En primer lugar quisiera anteponer a mis consideraciones de carácter comparativo sobre la crisis del parlamentarismo en Italia y España en los dos primeros decenios del siglo $x x$, algunas palabras de explicación acerca de la comparación, o mejor dicho del tipo de comparación que me propongo realizar. No queriendo demorarme demasiado sobre el tema, declaro de entrada que considero la brújula de toda comparación eficaz, el criterio que expresaba Tocqueville, cuando en L'Ancien régime et la révolution escribía que nadie que conociese sólo Francia y su historia, podía entender la gran revolución de $1789^{\prime}$. Con esto quiero decir que, mientras que tengo la mayor reserva hacia cierto tipo de comparación muy difuso que se presenta como una definición de fenómenos o procesos de carácter general -ya que muy a menudo se trata de una pseudocomparación al servicio de tesis predeterminadas-, la comparación me parece en cambio, no sólo útil sino necesaria, si apunta a un problema concreto, si se propone el mejor entendimiento de un fenómeno que se presenta en un determinado territorio, de escala regional o nacional, y que, en una primera aproximación, presenta analogías con fenómenos que se dan o se han dado en otros territorios de la misma escala.

Ajustándolo al tema que voy a tratar, el criterio de Tocqueville podría traducirse en el sentido de que no se puede entender la crisis del parlamentarismo en España, o en Italia, limitándose a analizar la crisis del parlamentarismo español $o$ italiano. $Y$ que por lo tanto el entendimiento de cada uno de esos fenómenos puede, y casi diría que debe, valerse de la mutua comparación. Obviamente esto no da por descontado que se trate de variantes nacionales de un mismo fenómeno, porque la utilidad de la comparación deriva tanto de las analogías como de las diferencias,

' Tocoueville, A. de, L'ancien régime et la Révolution, trad. it. Torino 1969, pág. 624 
como sabía perfectamente Tocqueville, el cual consideraba indispensable la comparación, aun sabiendo que no sólo la revolución francesa fue un fenómeno singularísimo, sino que también el sistema francés del antiguo régimen en que había surgido tenía sus muchas peculiaridades.

\section{COMPARACIÓN: SIMILITUDES Y DIFERENCIAS}

Llegando ahora a examinar la crisis del sistema parlamentario en Italia y España en aquella época, creo que las principales analogías que inducen a la comparación son, por un lado, la insuficiente representatividad de los parlamentos; por otro, las desviaciones en la actividad de sus miembros; y finalmente, el hecho de que en ambos países la crisis desembocó en una dictadura. Sin embargo, dentro de esas analogías hay diferencias que en definitiva son las que trazan la fisionomía particular de cada proceso y que la comparación, a través del contraste, ayuda a detectar. Incluso por lo que respecta a la desembocadura de ambas crisis en la dictadura, que es el elemento que, también por la contigüidad cronológica de los hechos, más nos da la impresión de encontrarnos ante fenómenos similares y paralelos, las diferencias no son pequeñas y creo que a partir de ellas podemos remontarnos a diferencias mayores que definen las peculiaridades de los dos casos.

Al hablar de distinciones entre las conclusiones de la crisis, quiero en primer lugar contrastar la idea de una relación de dependencia, aunque sea indirecta, entre el primero de los acontecimientos y el segundo, sugerida por la mayor importancia, tristemente modélica, de la dictadura fascista con respecto a la de Primo de Rivera. Porque ésta es en mi opinión una inferencia arbitraria fundada en una anticipación a los años 1922-1923 de un fenómeno que se desarrolla en los años sucesivos. No hay que olvidar - como parecen haberlo olvidado algunos que han enfatizado demasiado el precedente mussoliniano en relación a la dictadura de Primo de Rivera-, que en aquellos años en Italia todavía no hay una verdadera dictadura, ya que hasta después del delito Matteotti, es decir hasta 1925, siguen existiendo en la legalidad los partidos de oposición, incluso el comunista, que tienen una consistente minoría en el Parlamento ${ }^{2}$.

Cuando en España se da el golpe incruento de Primo de Rivera, en Italia todavía no existe un régimen fascista. $Y$ si bien las consecuencias

2 Sobre la fase anterior a la transformación del fascismo en régimen el libro básico sigue siendo el de Lyttelton, A., La conquista del potere. Il fascismo del 1919 al 1929. Bari 1974. 
de la marcia su Roma habían creado cierto clima internacional y sabemos que en algunos medios de la derecha española el ejemplo del fascismo italiano despertó entusiasmos y sugestiones ${ }^{3}$, para el golpe de Primo de Rivera no hizo falta ningún ejemplo extranjero, ya que enlazaba con la tradición española de los pronunciamientos. Una tradición que en Italia era absolutamente inexistente; más bien la tradición era exactamente la contraria - y se trata de un rasgo que en la comparación debe de aparecer en lugar eminente en el apartado de las diferencias-; es decir, que había una tradición de retraimiento de los militares de la política y de subordinación al poder civil, así que en el golpe mussoliniano de octubre de 1922, el papel de los militares fue totalmente marginal. En cambio, el de Primo de Rivera fue no sólo obviamente un golpe militar sino también la culminación de una vuelta a la intervención militar en la política que había empezado a partir, por lo menos, de 1917, y había continuado, en términos musicales, en crescendo.

Podría, sin embargo, suponerse que la toma del poder por parte de Mussolini y de Primo de Rivera respondiese, salvadas algunas diferencias, a una misma necesidad. No obstante, aun bajo este perfil, existen notables diferencias entre los dos casos, que se plasman en un distinto papel de las masas en el curso de las crisis y que remiten también a un diverso grado de representatividad de los parlamentos.

La historiografía italiana sobre el período que precede a la marcia su Roma ha valorado diversamente la importancia del efectivo peligro representado por el movimiento de las masas para la supervivencia del sistema de poder de las clases dominantes ${ }^{4}$. Pero todos los historiadores coinciden sobre el hecho de que - fuese un peligro real o por el contrario sólo un fantasma - la existencia de un poderoso movimiento de masas jugó un papel decisivo en la crisis del régimen liberal y parlamentario. Fue la existencia de este movimiento de masas - que pudo contar con partidos y sindicatos fuertemente organizados, no sólo de la izquierda, sino también católicos - el que, haciendo de alguna forma imprescindible la adopción del sistema electoral proporcional, aumentó enormemente la representatividad del Parlamento, pero al mismo tiempo despertó en la gran burguesía y gran parte de las clases medias graves temores.

Pero al margen de este crecimiento de la representatividad del Parlamento, que en efecto tuvo una incidencia bastante marginal en el de-

\footnotetext{
${ }^{3}$ Vid. a ese propósito, TUSELL, J. y AVILÉS, J., La derecha española contemporánea. Sus orí. genes: el maurismo. Madrid 1986.

${ }^{4}$ Para una valoración de las diversas tesis historiográficas vid. la obra de VIVARELLI, R., Storia delle origini del fascismo. Bologna 1991.
} 
sarrollo de la crisis italiana, fue el movimiento de las masas quien protagonizó el período. Los motines en contra de la carestía de la vida, las ocupaciones de tierras, especialmente - aunque no sólo- de los latifundios, las masivas huelgas campesinas y obreras - que llegaron a un máximo de casi 2.000 en 1920, implicando alrededor de 2 millones y medio de huelguistas-, y finalmente la ocupación masiva de las fábricas del norte en el otoño de 1920 en que culminó el llamado biennio rosso son los supuestos de la preguerra civil desencadenada por el squadrismo fascista y sin los cuales no se puede entender la destrucción del régimen liberal parlamentario en Italia.

En cambio, en el caso de España no se puede hablar en aquella misma época de un real peligro de las masas. Porque si bien la presencia de las masas en la escena social y política fue siempre más consistente y algunos momentos, como la huelga general de 1917 o la guerra sindicalista en Barcelona, pudieron asustar y aumentar la intolerancia de las capas burguesas, lo que parece más determinante son dos factores complementarios, es decir el desprestigio de la institución parlamentaria $-y$ más en general de toda la llamada «vieja política»- y la intervención militar.

Desprestigio e intervención militar son complementarios porque sin el primero la segunda hubiese sido por lo menos más difícil; y no sólo porque habría encontrado en la sociedad civil más resistencias de las que encontró - que como sabemos fueron nulas - sino también porque a los militares les hubiera faltado aquella autolegitimación que procedía de la convicción, compartida por todas las clases sociales, de la necesidad de limpieza y seriedad en la administración y la política. Ahora bien, no quiero decir que ésta fue la razón principal de su intervención; probablemente las razones circunstanciales más poderosas fueron las responsabilidades de Annual y la oportunidad de hacerse con el poder. Pero no hay por qué dudar de que también los militares aspirasen a un sistema de gobierno más limpio y serio. Esto no quiere decir que no padeciesen, como decía Ortega, igual que los abogados, los ingenieros, y en general todos sus compatriotas, aquella suerte de esquizofrenia por la que, aspirando a la anulación del caciquismo en la administración y la política, ellos mismos actuaban como caciques en su medio ambiente ${ }^{5}$. Pero esto es per-

\footnotetext{
${ }^{5}$ En su conocido artículo Sobre la vieja politica, Ortega escribia: “La economía de los particulares adolece de los mismos vicios que la finanza pública. La incompetencia del ministro y del parlamentario, su arbitrariedad, su caciquismo, reaparecen en el ingeniero, en el industrial, en el agricultor, en el catedrático, en el médico, en el escritor. Por eso es el mayor quid pro quo que cabe cometer imaginarse el caso de España como el de un pais donde una sociedad sana sufre los vicios y errores de unos cuantos gobernantes, de suerte que bastaría con des-
} 
fectamente compatible con el hecho de que estuvieran sinceramente hartos de gobiernos y parlamentos, gravemente desprestigiados tanto por su origen como por su actuación.

Hablar sobre el tema de la crisis del parlamentarismo supone por lo tanto analizar el elemento básico de esta crisis, es decir, el desprestigio del Parlamento en la opinión. Y en la raíz de este desprestigio estaba primordialmente el mismo origen electivo de las Cámaras. Aun bajo este aspecto nos encontramos ante notables diferencias entre el caso español y el italiano; y no sólo porque en vísperas del desenlace de la crisis italiana -que fue principalmente una crisis social más que política y parlamentaria - en el Parlamento, gracias a la aplicación del sistema proporcional, había una mayor coincidencia entre "país real» y "país legal». También si se examina todo el período de vigencia del sistema liberal y parlamentario en los dos países, la práctica electoral que engendra los Parlamentos es distinta, sobre todo si se considera el conjunto de ambos países.

\section{LAS RAZONES DEL DESPRESTIGIO: LAS ELECCIONES}

Ya he tratado este tema en un artículo ${ }^{6}$ y quisiera ser sintético, para dedicar luego más espacio a otro aspecto, es decir, el de la actuación concreta de los diputados. Por lo que se refiere a las elecciones, si nos atenemos a los testimonios y las denuncias de la época, que posteriormente han encontrado un eco en el juicio de gran parte de la historiografía, el caso de Italia no se nos presenta muy distinto del español.

Por ejemplo, en la Storia d'Italia Einaudi, Ernesto Regionieri, a propósito de las elecciones de 1892, ha escrito que:

"Giolitti hizo las elecciones políticas como ninguno de los anteriores gobiernos italianos, extremando y perfeccionando todos los instrumentos de intervención y presión anteriormente experimentados"?.

Y posteriormente, refiriéndose a las elecciones de 1913, las primeras con sufragio universal, también presididas por Giolitti, escribe:

terrar a éstos para que las virtudes nacionales den su lucida cosecha" ( $E /$ Sol, 27 de noviembre de 1923).

${ }^{6}$ RANZATo, G., "La forja de la soberanía nacional: las elecciones en los sistemas liberales italiano y español", en Ayer, núm. 3, 1991.

${ }^{7}$ Storia d'Italia Einaudi, vol. 4, t. III, pág. 1800. 
“Los métodos tradicionales de corrupción y de presión de los órganos periféricos del Estado dieron lugar, particularmente en la Italia meridional, pero más o menos en todas las áreas rurales más atrasadas, a una sólida campaña de intimidaciones y violencias en la que el aparato del Estado alternábase con la intervención activa de bandas de matones y de la mafia»" ${ }^{8}$.

Lo que importa destacar es por lo tanto que, lejos de haberlos inventado Giolitti, "los métodos de corrupción y de presión de los órganos periféricos del Estado», eran, como dice Ragionieri, “tradicionales», y que el hombre de Estado piamontés no había hecho más que extremar y perfeccionar «todos los instrumentos de intervención y presión anteriormente experimentados".

Por otra parte, la afirmación de Ragionieri se fundamentaba en múltiples e ilustres testimonios. Pasquale Turiello y Gaetano Mosca, por citar sólo algunos de los más destacados, han dejado páginas muy significativas sobre la práctica de adulteración del voto y de intervención gubernamental en las elecciones. Del periodo giolittiano son conocidas las ásperas denuncias de la corrupción electoral por parte de Gaetano Salvemini, que en su conocido pamphlet dedicado a Giolitti, que lleva el significativo título /l ministro della malavita, escribía:

«El señor Giolitti seguramente no es el primer hombre de gobierno de la Italia unida que considere el Mezzogiorno como tierra de conquista (...). Pero nadie ha sido tan brutal, tan cínico, tan falto de escrúpulos como él (...); nadie ha empleado tan sistemática y descaradamente, en las elecciones del Mezzogiorno, todo tipo de crímenes y violencias" ${ }^{9}$.

Para probar sus acusaciones Salvemini transcribía varios documentos entre los que destaca la carta enviada a un candidato en la proximidad de las elecciones por parte del alcalde de un pueblo pullés, que así le escribía:

«El prefetto Caracciolo ha sido muy débil y me ha auxiliado mucho, especialmente en el trabajo de eliminación. Sin embargo le veo algo escrupuloso a propósito de los medios que yo le propongo para conseguir el fin común. iSería oportuna, urgentísima, la disolución del ayuntamiento de Canosa!... Infundiria un verdadero terror y el triunfo sería asegurado (...). Se precisan delegados decididos e incondicionales. Se necesita la

8 Ibidem, pág. 1958.

${ }^{9}$ Salvemini, G., I/ ministro della malavite. Milano 1962 (1. a ed., 1910), pág. 138. 
renovación inmediata del sargento de los carabinieri de Ruvo, y tantas otras cosas que el prefetto puede acordar sin más trámites" ${ }^{10}$.

El documento transcrito por Salvemini ilustra una realidad que resulta muy familiar, hasta en la terminología - disolución de ayuntamientos, delegados, etc. - para los que conocen las vicisitudes electorales de la España de este mismo período. Sin embargo, si en la comparación pasamos del ámbito de los casos específicos, de las denuncias y de los testimonios, al ámbito de los datos cuantitativos, nos encontramos ante una relevante diferencia. De hecho, asumiendo como indicador de la extensión de las adulteraciones electorales, el número de las impugnaciones de elecciones por fraude que, en ambos países, llegaron a los organismos llamados a fallar sobre dichas impugnaciones, en el período que va de 1904 a 1914 - período que comprende los comicios italianos de 1904, 1907 y 1913 y los españoles de 1907, 1910 y 1914- nos encontramos con que el número de las elecciones fraudulentas -es decir, aquellas en que para el resultado fueron determinantes efectivas violencias, trampas y fraudesfue mucho menor en Italia que en España, además de estar concentradas en gran medida en el Mezzogiorno. Y esto a pesar de que el período elegido para la comparación es para Italia la época giolittiana, es decir, la que bajo el perfil de la probidad electoral tiene la peor fama.

De hecho en los tres comicios italianos las elecciones impugnadas fueron siempre menos de la décima parte de los escaños, mientras que en los españoles se va de la cuarta parte hasta lindar la mitad en los de 1914. Descontando cierta proporcionalidad entre elecciones impugnadas y las efectivamente fraudulentas, la indicación no podría ser más clara.

Por otra parte una convalidación de la veracidad de esta indicación la encontramos también, por lo que se refiere a Italia, en un testigo digno de crédito, es decir el mismo Gaetano Salvemini, que, ante la proximidad de las elecciones de 1913, en un artículo publicado en L'Unitá, escribía:

"[El gobierno] concentra sus fuerzas sólo en aquellos puntos en donde le es preciso hacer triunfar un servidor del ministerio y aplastar el candidato de la oposición. No son nunca más de un par de docenas de distritos en todo el Mezzogiorno" "11.

La mera consideración de los datos cuantitativos llevaría por lo tanto a la conclusión de que las elecciones en Italia fueron, a pesar de su fama,

10 Salvemini, G., ibidem, págs. 129-130.

1 L'Unità, 8 de agosto de 1913. 
mucho más correctas que en España. Sin embargo, sobre todo si nos ceñimos a las del Mezzogiorno, la desproporción abarca sólo las elecciones fraudulentas que las fuentes utilizadas documentan. Lo cual no implica que las elecciones italianas fuesen modélicas. Lo que caracteriza el caso italiano con respecto al español es el grado de incidencia del voto clientelar, es decir el voto conseguido a cambio de favores, que sobre todo en el Mezzogiorno fue la casi totalidad del voto que se emitía.

Ahora bien, a pesar de ser objeto de general reprobación, una notable proporción de voto clientelar no sólo está presente en las elecciones de todos los países, sino que se puede considerar consustancial al sistema liberal-democrático, un sistema en que, más allá de los principios, va sobreentendido que la mayoría de los electores votan no teniendo claras ideas sobre cómo y por qué se debe gobernar un país, y por lo tanto se dejan guiar por intereses de corto o mediano alcance. Lo peculiar de la España de este período es más bien una relativa baja incidencia de este tipo de voto. Cosa que por otra parte iba implícita en la misma regla del turno, cuya constancia en el funcionamiento no podía fundarse más que en el fraude.

La razón de fondo de este mayor peso del fraude en España y por lo tanto el mayor desprestigio del sistema parlamentario, estribaba básicamente en una menor disponibilidad de recursos de parte de los que competían para los escaños de diputado y sobre todo de parte de los gobiernos; lo cual impedía el funcionamiento en gran escala de aquel mecanismo que funcionaba en las elecciones italianas, es decir el intercambio de favores y beneficios con votos.

En la España de aquel entonces esos beneficios eran la meta de la contienda electoral, y por lo tanto no se podían derrochar para conseguir un poder político que, de tal forma hubiese quedado en gran parte vacío de recursos. Por otro lado, esa limitación de recursos hacía también precisa para la estabilidad del sistema, la alternancia en su aprovechamiento. De ahí la necesidad del turno, y otra vez del fraude, para vencer las resistencias, tanto de las clientelas que no querían aceptar la alternancia como, en menor medida, de aquella parte del electorado constantemente excluida del reparto.

Los gobernantes españoles no tenían tantas posibilidades como los italianos de construir carreteras, puentes, escuelas, cuarteles, etc.; o de procurar créditos, descontar impuestos, o crear muchos empleos, para conseguir votos. Por eso los Silvela, los Canalejas, los Maura, etc., a menudo al margen de su voluntad subjetiva, fueron condenados sin remedio por la escasez, a jugar sólo el papel de grandes sacerdotes de la regla del turno. 


\section{LAS RAZONES DEL DESPRESTIGIO: LA PRÁCTICA DE LAS CLIENTELAS}

El objetivo principal de la contienda electoral en España no era por lo tanto contribuir a legislar o a orientar el gobierno del país sino sobre todo el aprovechamiento de la administración. Esto por un lado complementaba el desprestigio del que adolecía la institución parlamentaria, ya que afectaba no sólo el origen sino también los fines de los miembros del Parlamento, pero por otro lado dependía de una idea y una práctica muy difusas en la relación existente entre política y administración.

De hecho las peculiaridades del caso español no se pueden entender fuera del marco del funcionamiento de una máquina administrativa del Estado que actuaba más como una constelación de funciones privatizadas y personalizadas que como una entidad de derecho público. Así que sus servicios, más que servicios impersonales de organismos estatales, eran el resultado de negociaciones interindividuales. De manera que la actuación política se confundía con una gestión discriminada de la administración.

Un elemento concreto de esta realidad lo representaba el hecho de que la función del diputado, tan disputada en las elecciones y, sobre el papel, eminentemente política, estribaba principalmente en la intermediación entre intereses particulares y la administración que podía satisfacerlos. Así que mientras que los gobiernos guardaban reservadas las iniciativas legislativas sobre asuntos de importancia, los diputados dedicaban gran parte de su actuación a leyes que beneficiaban a grupos restringidos.

Si se recorren las actas del Congreso se ve, por ejemplo, que uno de los empeños más frecuentes de sus miembros fue la participación en los trabajos de comisiones que deliberaban en materia de construcción de carreteras y ferrocarriles, y sobre todo decidian las condiciones de las contratas y las subvenciones gubernamentales. Hay algunas cifras que muestran que en algunos años las leyes sobre estas materias absorbieron la mayor parte de la actividad legislativa. Por ejemplo en la legislatura 1899-1900 las leyes relativas a ferrocarriles y carreteras fueron 243 sobre un conjunto de 329 leyes aprobadas; en 1901 fueron 71 sobre 117; en 1908-1909 fueron 101 sobre 179 , etc. ${ }^{12}$.

El número de diputados que tenian intereses en compañías de ferrocarriles fue siempre tan elevado que el tema fue objeto, a partir de 1892,

12 Esos datos se obtienen consultando los índices del Diario de sesiones del Congreso de las leyes aprobadas en cada legislatura. 
de varios proyectos de ley que apuntaban a declarar la incompatibilidad entre el cargo de diputado y el de socio o dependiente de esas compañías o de otras contratadas por el Estado. En 1903, ilustrando su proyecto, el integrista Nocedal declaraba:

"La conciencia pública está alarmada y recelosa al ver la compatibilidad constante, crónica, endémica, que hay entre los cargos de diputado y de ministro, con los cargos de consejero de Administración y abogados permanentes y agentes de las empresas concesionarias de obras y servicios públicos o monopolios del Estado (...). Porque sucede que estas empresas tienen a sueldo y merced a muchos hombres importantes de la política, y ocurre que de resultas y con tan buenos padrinos las empresas hacen lo que quieren impunemente" ${ }^{13}$.

Otros proyectos de Salmerón, Canalejas y Alba, intentaron establecer esa incompatibilidad, pero todos quedaron en letra muerta ya que chocaban con intereses demasiado poderosos y consustanciales con una noción desviada pero dominante de la función de la administración y de la política.

No se debe, sin embargo, creer que la mayoría de los diputados estuviesen al servicio de grandes intereses. Si bien, en una visión estrecha de sus deberes, muchos de ellos cumplían una función de trámite entre intereses, a veces mínimos y a menudo lícitos, y los organismos de la administración central. La mayor parte de la actividad del diputado no se desarrollaba en las Cortes, sino en los ministerios, donde realizaban una obra de transmisión, solicitud, garantía, etc., de las más variadas instancias dirigidas a la administración por parte de individuos o grupos de intereses, que de otro modo hubiesen tenido pocas oportunidades de verse satisfechas.

En realidad la extremada centralización administrativa hacía casi indispensable esa intermediación de casi toda iniciativa que se saliese de la estrecha esfera de lo privado. En la casi total ausencia del Estado de derecho, la vía maestra, el conducto casi obligado entre ciudadano y administración central, era el diputado. Es decir, que gran parte de los servicios y las funciones realizadas impersonalmente en un Estado de derecho, se personalizaban y concedían con parcialidad en un área de clientelas sobre todo gracias a sus trámites.

En realidad en aquel entonces era considerado normal que ésta fuera la principal función del diputado. La prensa daba normalmente noticia de

${ }^{13}$ Diario de sesiones del Congreso, 30 de junio de 1903, pág. 641. 
la actividad ministerial de los representantes en Cortes. En el anuario parlamentario de Sánchez de los Santos de 1910, por ejemplo, se lee este perfil del diputado por Mérida Carlos Pacheco:

"No tardará en aumentar su significación y su influencia entre sus electores, no sólo con la labor callada y continua en favor de ellos en los Ministerios, sino en la acción parlamentaria y pública» ${ }^{14}$.

Parece como si «la acción parlamentaria y pública» fuera una función accesoria y la fundamental fuese "la labor en los Ministerios".

\section{FRONTERAS LÁBILES: POLÍTICA Y ADMINISTRACIÓN}

El contexto en que se desarrollaba esa labor de los diputados y que caracterizaba particularmente el caso español es evidentemente el de una confusión y casi indistinción entre los agentes de la política y los de la administración. Así que ni los segundos estaban claramente subordinados a los primeros, ni la discrecionalidad del político trasladada al campo administrativo se advertía claramente como ilícita.

Esta peculiaridad del caso español adquiere el máximo relieve si se observa la extrema intercambiabilidad y confusión entre personal político y personal administrativo que abarcaba prácticamente todas las ramas de la administración. En primer lugar tenemos el ejemplo de los gobernadores civiles. El estudio de Bernard Richard ha puesto de relieve el origen prevalentemente político de los titulares de aquel cargo, ya que el 68 por 100 de los que lo ocuparon entre 1874 y 1923, resultan haber sido anteriormente diputados provinciales, diputados a Cortes o senadores ${ }^{15}$. Pero no fue tanto ésta la particularidad del caso español - ya que prefetti de origen político hubo, si bien en menor medida, tanto en Italia como en Francia - sino más bien el fenómeno inverso absolutamente insólito en los otros países, es decir, el de diputados que procedian de las filas de los gobernadores civiles. Por ejemplo en las Cortes de 1910 había 33 diputados que habían ocupado el cargo de gobernador ${ }^{16} ;$ y gobernadores fueron no pocos ministros y políticos de primera fila, como González Besada, Ruiz Jiménez y Juan de la Cierva.

14 Sánchez de los Santos, M., Las Cortes españoles de 1910. Madrid 1911, pág. 565.

15 RICHARD, B., "Etude sur les gouverneurs civits en Espagne de la Restauration à la dictature (1874-1923)", en Mélanges de la Casa de Velázquez, 1972, págs. 448 y ss.

${ }^{16}$ El dato se deduce del examen de los perfiles biográficos de los diputados publicados en la citada obra de M. Sánchez de los Santos. 
El de los gobernadores civiles no es un ejemplo aislado sino uno de los muchos que muestran la casi absoluta confusión de personal político y personal administrativo que abarcaba prácticamente todas las ramas de la administración española. Lo más significativo a este respecto es el hecho de que, si bien la ley de 1880 sobre incompatibilidades parlamentarias limitaba a una restringida clase de funcionarios públicos la posibilidad de acceder a las Cortes, ésta se fue ampliando a través de interpretaciones extensivas de la ley y normas complementarias. Y sobre todo, aunque la ley - al igual que la ley italiana sobre incompatibilidades parlamentarias- fijó un techo de 40 miembros de la administración pública que podrían sentarse en el Congreso, este número fue a menudo ampliamente superado.

A pesar de las medidas encaminadas a limitar los abusos y de las denuncias periódicamente pronunciadas en el mismo recinto de las Cortes, "la ley de incompatibilidades, que dependía para su aplicación de la buena voluntad del Gobierno y de la mayoría, es decir de los posibles afectados de la misma, se tornaba precisamente por ello mismo en una norma huera cuya utilidad práctica era inexistente" ${ }^{17}$. De ahí la crecida dimensión de la violación de esa ley. Así que, por ejemplo, en las Cortes de 1901 los dependientes de la administración pública eran 87, y en las de 1918 eran 108, es decir más de la cuarta parte de los diputados ${ }^{18}$. Y entre ellos generalmente estaban los más altos cargos de la administración del Estado, como, por ejemplo, el Fiscal del Supremo, el Presidente del Tribunal de Cuentas y muchos Directores generales de varios ministerios.

También en este caso funcionaba la posibilidad del pasaje inverso; es decir que el sólo requisito de ex-diputado podía bastar para acceder a los cargos de la administración. El fenómeno fue tan extenso que también en este caso se presentaron varios proyectos de ley para eliminarlo, a partir del presentado por García Alix en 1891, en cuyo preámbulo se leía:

«Desde hace bastante tiempo la opinión pública por sus órganos y medios de manifestación, se ha pronunciado en el sentido de evitar que los altos puestos de la administración se ocupen sin más títulos ni méritos

\footnotetext{
17 Martinez Sospedra, M., Incompatibilidades parlamentarias en España (1810-1936). Valencia 1974 , pág. 143

${ }^{18}$ Los datos se deducen del examen de las Actas parlamentarias cotejando los elencos enviados en fases sucesivas a la Junta de incompatibilidades por los ministerios, los fallos de la misma Junta y la concreta participación de los funcionarios elegidos en las discusiones de las Cortes.
} 
que aquellos que nacen de la elección para Diputados a Cortes», y cuyo artículo único decía:

«El cargo de Diputado, por sí solo y sin que concurran en la persona que lo desempeña las condiciones de carrera y servicio del Estado exigidas por la ley (...) no dará derecho para obtener ningún destino en la Administración Pública» ${ }^{19}$.

Sucesivamente también Francisco Silvela introdujo en un proyecto de ley relativo a toda la materia de las incompatibilidades una norma que tenía el mismo objeto. En su preámbulo se leia:

«Se ha unido a este proyecto un precepto que quizá se estimará por algunos un tanto ajeno a su propia materia, en el que se excluye para lo por venir el cargo de Diputado o Senador de las condiciones de aptitud para las funciones administrativas; pero no es ciertamente ajeno a la finalidad de la ley, que no es otra que la de contribuir a la efectiva separación de las funciones administrativas de la vida y acción del Parlamento" ${ }^{20}$.

Pero era imposible, a través de una simple iniciativa legislativa, aunque fuese respaldada por el prestigio de un Silvela, eliminar una práctica que a fin de cuentas resumía el sentido de todo el sistema. Así que ni su proyecto, ni otros parecidos, se convirtieron en ley. $Y$, si bien la especialización de algunas tareas administrativas y la implicita creación de cuerpos de funcionarios de carrera limitaron el fenómeno de los nombramientos políticos en puestos que exigían una concreta capacitación, el turno se mantuvo vigente también por lo que se refiere al acceso a los cargos de la administración. Lo cual quiere decir que se mantuvo el turno en el aprovechamiento de las oportunidades para las clientelas logradas por el control de la máquina administrativa.

\section{COMPATIBILIDADES ENTRE CLIENTELISMO, CORRUPCIÓN Y "GRANDE POLITICA»}

Aunque sea oportuno subrayar esta importante diferencia entre los dos países que estamos comparando - ya que en Italia la administración estuvo mucho más separada de la política, lo cual no quiere decir que fuese independiente-, no es sin embargo la sola personalización, cuando no la corrupción, de la administración pública la que pueda explicar -sobre

${ }^{19}$ Diario de sesiones de las Cortes, Ap. 25 al núm. 43 del 27 de abril de 1891.

${ }^{20}$ Diario de sesiones de las Cortes, Ap. 1 al núm. 71, del 25 de noviembre de 1899. 
todo si no pasa de cierto nivel de guardia - la crisis de desprestigio y el fracaso de todo un sistema político-administrativo, como el que indudablemente padeció el español, y que desembocó en la dictadura. Es sabido que también en el sistema italiano prosperaba la corrupción. El denominado affarismo de los diputados italianos fue objeto de denuncias constantes. A los diputados de las Cámaras de Giolitti, que debían en gran medida el cargo a sus favores, se les llamaba los ascari; es decir, que se les aplicaba el nombre de las tropas indígenas de las colonias, tropas de incondicionales a sueldo. Y el sueldo, en el caso de los diputados, era la satisfacción de los apetitos de las clientelas locales.

El ejemplo más evidente de que cierto nivel de corrupción no puede llevar por sí mismo a la disolución de un sistema liberal es el de Estados Unidos, más o menos en este mismo período, es decir entre finales del siglo xIX y los primeros decenios del siglo xx. El aprovechamiento de la administración pública para intereses particulares acaso en ningún país ha llegado a niveles tan altos, no sólo porque es allí donde se ha inventado el spolls system, sino por el grado de franqueza, hasta la desfachatez, con que muchos candidatos se declaraban al servicio de intereses que no tenian nada que ver con el bien de la nación. $Y$ algunos hasta llegaron a teorizar la legitimidad de sus mismos intereses personales. En este sentido el caso más significativo es el del diputado George Washington Plunkitt del distrito de Nueva York que en muchos discursos y escritos mantuvo la conveniencia del interés personal del diputado, distinguiendo entre concusión honesta y deshonesta. En un famoso artículo publicado en 1905 por ejemplo, refiriéndose a su partido escribia:

«Sí, es cierto, muchos de nuestros hombres se han hecho ricos con la política. Entre ellos yo mismo. He acumulado una gran fortuna, y cada día me vuelvo más rico, pero nunca he practicado la concusión deshonesta - es decir chantajear a tablajeros, rufianes, etc.-, ni lo han hecho los demás que en virtud de la política han reunido grandes capitales. Existe una concusión honesta y yo soy un ejemplo viviente de cómo funciona.

Dejadme explicar con un ejemplo. Mi partido está en el poder y está a punto de emprender unas obras públicas, por ejemplo, la construcción de un nuevo parque. Yo veo la buena ocasión y la cojo al vuelo. Me precipito a comprar cuantas más tierras puedo en la zona. Luego, cuando la administración hace público el proyecto, se abre la carrera a la compra de esas tierras que antes no interesaban a nadie. ¿No es perfectamente honesto exigir un buen precio y realizar una ganancia sobre mi inversión y mi buena vista? Sí que lo es, y ésta es la que llamo concusión honesta" 21.

${ }^{21}$ Cit. en Riordon, W. L., Plunkitt of Tammany Hall, ed. it. Pise 1991, págs. 37-38. 
Se trata de un caso que por no ser aislado, y en cualquier caso por ser público y teniendo como protagonista un diputado que representó a sus electores en el Congreso de Estados Unidos durante quince años, es muy significativo. $Y$ es muy significativo que, a pesar de la amplia difusión en aquella época de este sistema de los bosses y de la machine politics, el régimen liberal de Estados Unidos nunca peligró, como nunca peligró en Italia en la época de Giolitti. La razón de esto estriba básicamente en el hecho de que en estos países esa política personalista, clientelista y hasta corrupta, no impidió y hasta llegó en algunos momentos a ser funcional a equilibrios que permitían el desarrollo de una política de grandes vuelos.

Aunque la mayor independencia del ejecutivo con respecto al legislativo prevista en el sistema constitucional americano dejara más espacio a los presidentes para realizar una política menos influida por los pequeños intereses representados por los diputados, es indudable que no fue al margen del Congreso como se realizó en Estados Unidos, a partir de los últimos dos decenios del siglo xIx, una política que favoreció la gran expansión económica y consecuentemente imperialista de aquel país. Igualmente y más aún la política de Giolitti dirigida a realizar el despegue industrial de Italia y al mismo tiempo una mayor integración nacional de las masas populares a través de una legislación social que las beneficiara, tuvo como pieza básica esas Cámaras dóciles integradas por los ascari satisfechos con el logro de sus objetivos clientelares.

Esto es lo que marca la diferencia entre España e Italia. Es decir, que en España la gran política fue atropellada y hecha inviable por la lógica de las clientelas. La lógica de las clientelas presidía el turno, y el turno impedía cualquier política de grandes vuelos. Si no por otra cosa, por la brevedad de permanencia en el poder de cualquier gobierno. Giolitti, con breves interrupciones, controló el poder durante quince años; en cambio el gobierno español de más larga duración en este período fue el denominado "gobierno largo" de Maura que duró dos años y pico. Es por esto por lo que es lícito preguntarse: ¿existió una política económica, existió una política social en España durante los dos primeros decenios del siglo $x x$ ? Es harto evidente que sólo existieron inevitablemente medidas fragmentarias, mientras que la única línea de continuidad fue la del spolls system identificada en la opinión con la política sin más, y capaz por lo tanto de desprestigiar toda la política y sus instituciones y dejar paso al golpe militar.

Por lo tanto, a pesar de tener un desenlace parecido, los dos casos, el italiano y el español, tienen una marcada diferencia. Porque sólo en el caso de España se puede hablar de una verdadera crisis del sistema 
parlamentario como fundamento de una crisis general. En cambio en el caso de Italia el Parlamento, aunque haya crecido en representatividad, queda al margen de un movimiento de masas que, si no llega a ser verdaderamente revolucionario, constituye una amenaza $o$, en cualquier caso, impide un tranquilo desarrollo de la sociedad burguesa, para cuya continuidad hacía falta un régimen reaccionario, pero capaz de implicar a las masas. De ahí el diabólico invento italiano, desgraciadamente exportado en muchas partes del mundo, que fue el régimen fascista. 